\title{
High expression of transcriptional coactivator p300 correlates with aggressive features and poor prognosis of hepatocellular carcinoma
}

Mei $\mathrm{Li}^{1,2+}$, Rong-Zhen Luo ${ }^{1,2+}$, Jie-Wei Chen ${ }^{1,2}$, Yun Cao ${ }^{1,2}$, Jia-Bin Lu ${ }^{1,2}$, Jie-Hua He $e^{1,2}$, Qiu-Liang Wu ${ }^{1,2}$, Mu-Yan Cai ${ }^{1,2^{*}}$

\begin{abstract}
Background: It has been suggested that p300 participates in the regulation of a wide range of cell biological processes and mutation of p300 has been identified in certain types of human cancers. However, the expression dynamics of p300 in hepatocellular carcinoma (HCC) and its clinical/prognostic significance are unclear.

Methods: In this study, the methods of reverse transcription-polymerase chain reaction (RT-PCR), Western blotting and immunohistochemistry (IHC) were utilized to investigate protein/mRNA expression of p300 in HCCs. Receiver operating characteristic (ROC) curve analysis, spearman's rank correlation, Kaplan-Meier plots and Cox proportional hazards regression model were used to analyze the data.

Results: Up-regulated expression of p300 mRNA and protein was observed in the majority of HCCs by RT-PCR and Western blotting, when compared with their adjacent non-malignant liver tissues. According to the ROC curves, the cutoff score for p300 high expression was defined when more than $60 \%$ of the tumor cells were positively stained. High expression of p300 was examined in 60/123 (48.8\%) of HCCs and in 8/123 (6.5\%) of adjacent nonmalignant liver tissues. High expression of p300 was correlated with higher AFP level, larger tumor size, multiplicity, poorer differentiation and later stage $(P<0.05)$. In univariate survival analysis, a significant association between overexpression of p300 and shortened patients' survival was found $(P=0.001)$. In different subsets of HCC patients, p300 expression was also a prognostic indicator in patients with stage II $(P=0.007)$ and stage III $(P=0.011)$. Importantly, p300 expression was evaluated as an independent prognostic factor in multivariate analysis $(P=$ 0.021). Consequently, a new clinicopathologic prognostic model with three poor prognostic factors ( $p 300$ expression, AFP level and vascular invasion) was constructed. The model could significantly stratify risk (low, intermediate and high) for overall survival $(P<0.0001)$.
\end{abstract}

Conclusions: Our findings provide a basis for the concept that high expression of p300 in HCC may be important in the acquisition of an aggressive phenotype, suggesting that p300 overexpression, as examined by $\mathrm{HCC}$, is an independent biomarker for poor prognosis of patients with HCC. The combined clinicopathologic prognostic model may become a useful tool for identifying HCC patients with different clinical outcomes.

\section{Background}

Hepatocellular carcinoma (HCC) is the fifth most common cancer in the world and the third leading cause of cancer mortality [1]. It is among the top three causes of cancer death in the Asian Pacific region due to the high

\footnotetext{
* Correspondence: caimuyan@hotmail.com

+ Contributed equally

'State Key Laboratory of Oncology in South China, Sun Yat-Sen University

Cancer Center, Guangzhou, PR China

Full list of author information is available at the end of the article
}

prevalence of chronic hepatitis $\mathrm{B}$ virus and hepatitis $\mathrm{C}$ virus infections, and recently its incidence in the United States and in Western Europe has been increasing [2,3]. Despite new therapies and attempts for early detection of primary HCC, the long-term survival of HCC patient remains poor. Surgery is considered as one of the standard curative treatments for $\mathrm{HCC}$ if the tumor is resectable [4]. However, the prognosis of HCC patients with the same clinical stage often differs substantially in spite

\section{Biomed Central}


of curative surgical resection and such large variation is mostly unexplained. Thus, a large amount of investigations on HCC have focused on the discovery of specific molecular markers that could serve as reliable prognostic factors. To date, however, the search for specific molecules in HCC cells that have clinical/prognostic value remains substantially limited.

Recently, it has been reported that p300, a member of the histone acetyltransferase family of transcriptional coactivator, is found to play a variety of roles in the transcription process and catalyzes histone acetylation through its histone acetyltransferase activity [5,6]. Transcriptional coactivator p300 has been shown to participate in the regulation of various cellular processes such as proliferation, differentiation, apoptosis, cell-cycle regulation and DNA damage response [7]. A tumor suppressor role of $\mathrm{p} 300$ has been identified in certain types of human cancers, including breast, colorectal and gastric carcinoma [8,9]. However, several studies suggest that transcriptional coactivator p300 is a positive regulator of cancer progression and related to tumorigenesis of various human cancers $[10,11]$. The translational coactivator p300 was found to be involved in the integrin beta-1-mediated histone acetylation and p21 transcriptional activation in HCC [12]. In addition, Wang et al [13] suggested that a direct role of phosphor-CREB in p300 and Brg I recruitment to the Hulc promoter led to the activation of epigenetic markers and chromatin remodeling at the same location in hepatic cancer cells. It has also been reported that p300 expression correlates with nuclear alterations of tumor cells and contributes to the growth of prostate carcinoma and is a predictor of aggressive features of this cancer [14,15].

Up to date, the clinicopathologic/prognostic implication of p300 in HCC has not been explored. In this study, reverse transcription-polymerase chain reaction (RT-PCR), Western blotting, immunohistochemistry (IHC) and tissue microarray were utilized to examine the distribution and frequency of p300 expression in our HCC cohort and adjacent non-malignant liver tissues. In order to avoid predetermined cutpoint, receiver operating characteristic (ROC) curve analysis was employed to define the cutoff score for high expression of p300. In addition, the correlation between p300 expression and cell proliferation levels in our HCCs was analyzed using the Ki-67 assessment marker.

\section{Methods}

\section{Patients and tissue specimens}

In this study, the paraffin-embedded pathologic specimens from 123 patients with HCC were obtained from the archives of Department of Pathology, Sun Yat-Sen University Cancer Center, Guangzhou, China, between July 2005 and May 2008. The cases selected were based on distinctive pathologic diagnosis of $\mathrm{HCC}$, undergoing primary and curative resection for tumor without preoperative anticancer treatment, availability of resection tissue and follow-up data. These HCC cases included $107(87.0 \%)$ men and 16 (13.0\%) women, with mean age of 47.7 years. Average follow-up time was 26.79 months (median, 28.0 months; range, 1.0 to 61 months).

Patients whose cause of death remained unknown were excluded from our study. Clinicopathologic characteristics for these patients including age, sex, hepatitis history, alpha-fetoprotein (AFP), liver cirrhosis, tumor number, size, differentiation, stage, vascular invasion and relapse were detailed in Table 1 . Tumor differentiation was based on the criteria proposed by Edmonson and Steiner [16]. Tumor stage was defined according to American Joint Committee on Cancer/International Union Against Cancer tumor-node-metastasis (TNM) classification system [17]. Institute Research Medical Ethics Committee of Sun Yat-Sen University Cancer Center granted approval for this study.

\section{RT-PCR}

Total RNA was isolated from 8 pairs of HCC tissues and adjacent non-malignant liver tissues using TRIZOL reagent (Invitrogen, Carlsbad, CA). RNA was reversetranscribed using SuperScript First Strand cDNA System (Invitrogen, Carlsbad, CA) according to the manufacture's instructions. PCR was performed as described previously using specific primers for p300 [18]. The expression of GAPDH was monitored as a control.

\section{Western blotting analysis}

Equal amounts of whole cell and tissue lysates were resolved by SDS-polyacrylamide gel electrophoresis (PAGE) and electrotransferred on a polyvinylidene difluoride (PVDF) membrane (Pall Corp., Port Washington, NY). The tissues were then incubated with primary mouse monoclonal antibodies against human anti-p300 (Abcam, Cambridge, MA) at a concentration of $0.5 \mu \mathrm{g}$ / $\mathrm{ml}$. The immunoreactive signals were detected with enhanced chemiluminescence kit (Amersham Biosciences, Uppsala, Sweden). The procedures followed were conducted in accordance with the manufacturer's instructions.

\section{Tissue microarray (TMA) construction}

Tissue microarray was constructed as the method described previously [19]. In brief, formalin-fixed, paraffin-embedded tissue blocks and the corresponding H\&E-stained slides were overlaid for TMA sampling. The slides were reviewed by a senior pathologist (M-Y. C.) to determine and mark out representative tumor areas. Triplicates of $0.6 \mathrm{~mm}$ diameter cylinders were punched from representative tumor areas and from 
Table 1 Correlation of p300 expression with patients' clinicopathologic features in primary hepatocellular carcinomas

\begin{tabular}{|c|c|c|c|c|}
\hline \multirow[b]{2}{*}{ Variable } & \multicolumn{4}{|c|}{ p300 protein } \\
\hline & $\begin{array}{l}\text { All } \\
\text { cases }\end{array}$ & $\begin{array}{l}\text { Low } \\
\text { expression }\end{array}$ & $\begin{array}{l}\text { High } \\
\text { expression }\end{array}$ & $\begin{array}{l}P \\
\text { value }^{\mathrm{a}}\end{array}$ \\
\hline Age (years) & & & & 0.267 \\
\hline$\leq 47.7^{b}$ & 59 & $28(47.5 \%)$ & $31(52.5 \%)$ & \\
\hline$>47.7$ & 64 & $35(54.7 \%)$ & $29(45.3 \%)$ & \\
\hline Sex & & & & 0.564 \\
\hline Male & 107 & 55 (51.4\%) & $52(48.6 \%)$ & \\
\hline Female & 16 & $8(50.0 \%)$ & $8(50.0 \%)$ & \\
\hline Etiology & & & & 0.295 \\
\hline HBV & 97 & $48(49.5 \%)$ & 49 (50.5\%) & \\
\hline $\mathrm{HCV}$ & 8 & $3(37.5 \%)$ & $5(62.5 \%)$ & \\
\hline None & 18 & $12(66.7 \%)$ & $6(33.3 \%)$ & \\
\hline $\operatorname{AFP}(\mathrm{ng} / \mathrm{ml})$ & & & & 0.000 \\
\hline$\leq 20$ & 68 & $46(67.6 \%)$ & $22(32.4 \%)$ & \\
\hline$>20$ & 55 & 17 (30.9\%) & $38(69.1 \%)$ & \\
\hline Liver cirrhosis & & & & 0.334 \\
\hline Yes & 87 & $47(54.0 \%)$ & $40(46.0 \%)$ & \\
\hline No & 36 & $16(44.4 \%)$ & $20(55.6 \%)$ & \\
\hline Tumor size (cm) & & & & 0.000 \\
\hline$\leq 5$ & 76 & $50(65.8 \%)$ & $26(34.2 \%)$ & \\
\hline$>5$ & 47 & $13(27.7 \%)$ & $34(72.3 \%)$ & \\
\hline Tumor multiplicity & & & & 0.012 \\
\hline Single & 85 & $50(58.8 \%)$ & $35(41.2 \%)$ & \\
\hline Multiple & 38 & $13(34.2 \%)$ & $25(65.8 \%)$ & \\
\hline Differentiation & & & & 0.036 \\
\hline Well & 15 & $12(80.0 \%)$ & $3(20.0 \%)$ & \\
\hline Moderate & 70 & 36 (51.4\%) & $34(48.6 \%)$ & \\
\hline Poor & 32 & $14(43.8 \%)$ & $18(56.3 \%)$ & \\
\hline Undifferentiated & 6 & $1(16.7 \%)$ & $5(83.3 \%)$ & \\
\hline Stage & & & & 0.015 \\
\hline । & 12 & $10(83.3 \%)$ & $2(16.7 \%)$ & \\
\hline$\|$ & 49 & 27 (55.1\%) & 22 (44.9\%) & \\
\hline III & 48 & $23(47.9 \%)$ & $25(52.1 \%)$ & \\
\hline IV & 14 & $3(21.4 \%)$ & $11(78.6 \%)$ & \\
\hline Vascular invasion & & & & 0.130 \\
\hline Yes & 55 & $24(43.6 \%)$ & 31 (56.4\%) & \\
\hline No & 68 & $39(57.4 \%)$ & $29(42.6 \%)$ & \\
\hline Relapse & & & & 0.182 \\
\hline Yes & 42 & $18(42.9 \%)$ & $24(57.1 \%)$ & \\
\hline No & 81 & 45 (55.6\%) & $36(44.4 \%)$ & \\
\hline Ki67 expression & & & & 0.002 \\
\hline Low & 68 & 44 (64.7\%) & $24(35.3 \%)$ & \\
\hline High & 50 & $18(36.0 \%)$ & 32 (64.0\%) & \\
\hline
\end{tabular}

${ }^{\mathrm{a}}$ Chi-square test; ${ }^{\mathrm{b}}$ Mean age; HBV, hepatitis B virus; $\mathrm{HCV}$, hepatitis B virus; AFP, alpha-fetoprotein.

adjacent non-malignant liver tissue of individual donor tissue block and re-embedded into a recipient paraffin block at defined position, using a tissue arraying instrument (Beecher Instruments, Silver Spring, MD).
The TMA block contained 126 HCCs and adjacent nonmalignant liver tissues.

\section{Immunohistochemistry (IHC)}

The TMA slides were dried overnight at $37^{\circ} \mathrm{C}$,deparaffinized in xylene, rehydrated through graded alcohol, immersed in 3\% hydrogen peroxide for 20 minutes to block endogenous peroxidase activity, and antigenretrieved by pressure cooking for 3 minutes in ethylenediamine tetraacetic acid (EDTA) buffer $(\mathrm{pH}=8.0)$. Then the slides were preincubated with $10 \%$ normal goat serum at room temperature for 30 minutes to reduce nonspecific reaction. Subsequently, the slides were incubated with mouse monoclonal anti-p300 (Abcam, Cambridge, MA) at a concentration of $3 \mathrm{ng} / \mathrm{ml}$ and mouse monoclonal anti-Ki-67 (Zymed Laboratories Inc., South San Francisco, CA, 1:100 dilution) for 2 hours at room temperature. The slides were sequentially incubated with a secondary antibody (Envision; Dako, Glostrup, Denmark) for 1 hour at room temperature, and stained with DAB (3,3-diaminobenzidine). Finally, the sections were counterstained with Mayer's hematoxylin, dehydrated, and mounted. A negative control was obtained by replacing the primary antibody with a normal murine IgG. Known immunostaining positive slides were used as positive controls.

\section{IHC evaluation}

Nuclear immunoreactivity for $\mathrm{p} 300$ protein was reported in semi-quantitative method by evaluating the number of positive tumor cells over the total number of tumor cells. Scores were assigned by using $5 \%$ increments $(0 \%$, 5\%, 10\%-100\%). Expression for p300 was scored by 3 independent pathologists (M. L., R-Z. L. and M-Y. C.) blinded to clinicopathologic data. Their conclusions were in complete agreement in $82.1 \%$ of the cases, which identified this scoring method as highly reproducible.

\section{Selection of Cutoff Score}

ROC curve analysis was employed to determine cutoff score for tumor "high expression" by using the 0,1criterion [20]. At the p300 score, the sensitivity and specificity for each outcome under study was plotted, thus generating various $\mathrm{ROC}$ curves (Figure 1). The score was selected as the cutoff value, which was closest to the point with both maximum sensitivity and specificity. Tumors designated as "low expression" for p300 were those with scores below or equal to the cutoff value, while "high expression" tumors were those with scores above the value. In order to use ROC curve analysis, the clinicopathologic features were dichotomized: AFP level $(\leq 20 \mathrm{ng} / \mathrm{ml}$ or $>20 \mathrm{ng} / \mathrm{ml})$, tumor size $(\leq 5 \mathrm{~cm}$ or $>5$ $\mathrm{cm}$ ), tumor multiplicity (single or multiple), tumor 
A.

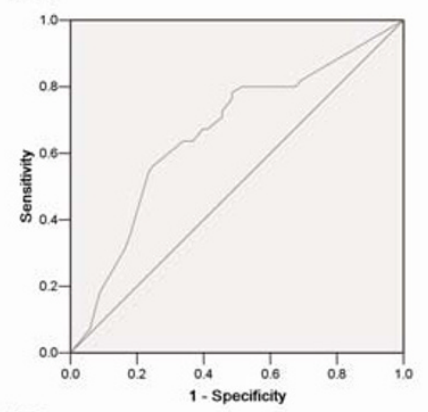

D.

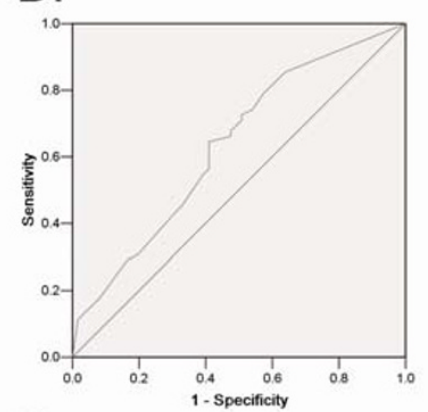

G.

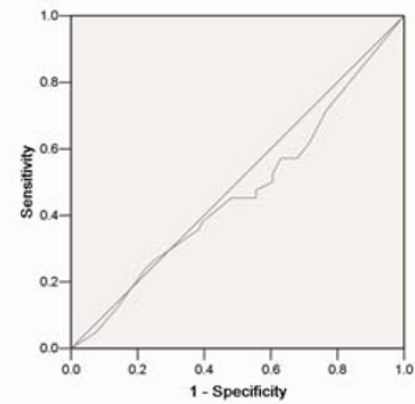

B.

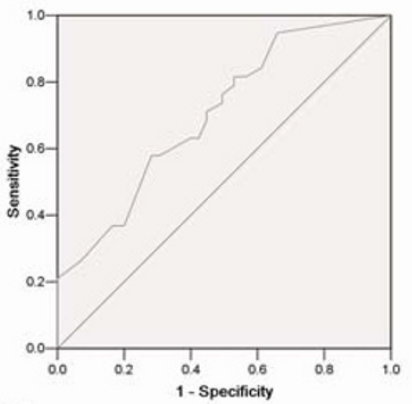

E.

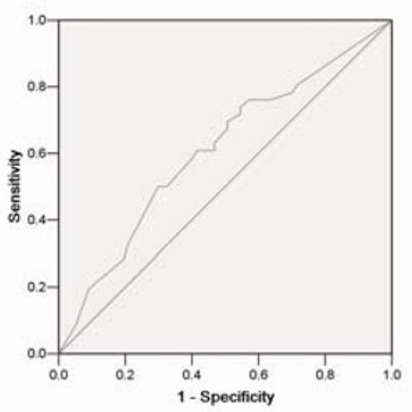

C.

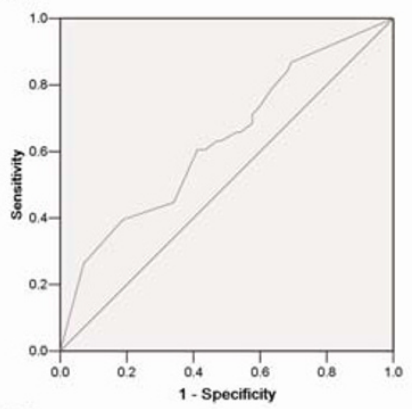

F.

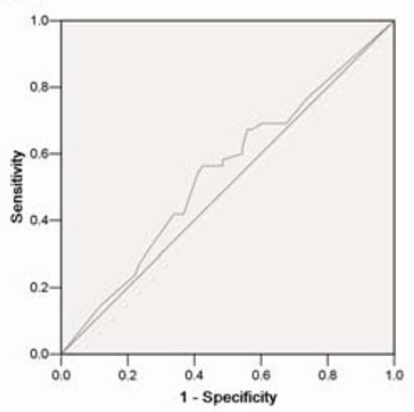

Figure 1 ROC curve analysis was created to determine the cutoff score for high expression of p300 protein. The sensitivity and specificity for each outcome were plotted: AFP level (A.), tumor size (B.), tumor multiplicity (C.), tumor differentiation (D.), clinical stage (E.), vascular invasion (F.), tumor relapse(G.).

grade (well-moderately or poorly-undifferentiated), stage $(\mathrm{I}+\mathrm{II}$ or III + IV), vascular invasion (absence or presence), relapse (absence or presence) and survival status (death due to HCC or censored).

\section{Statistical analysis}

Statistical analysis was performed by using the SPSS statistical software package (standard version 13.0; SPSS, Chicago, IL). ROC curve analysis was applied to determine the cutoff score for high expression of p300 and Ki67. The correlation between p300 expression and clinicopathologic features of HCC patients was evaluated by $\chi^{2}$-test. Univariate and multivariate survival analyses were performed using the Cox proportional hazards regression model. Survival curves were obtained with the Kaplan-Meier method. Predictive accuracy was quantified using the Harrell concordance index. Differences were considered significant if the $P$-value from a two-tailed test was $<0.05$.

\section{Results}

p300 mRNA expression examined by RT-PCR and p300 protein expression by Western blotting in liver tissues In this study, the status of expression of $p 300$ mRNA and p300 protein was further examined by RT-PCR and Western blotting, respectively, in 8 pairs of fresh HCC and adjacent non-tumorous liver specimens. The results showed that a total of $5 / 8(62.5 \%)$ HCCs was examined as having up-regulated $p 300$ mRNA expression, when compared with their adjacent non-malignant liver 


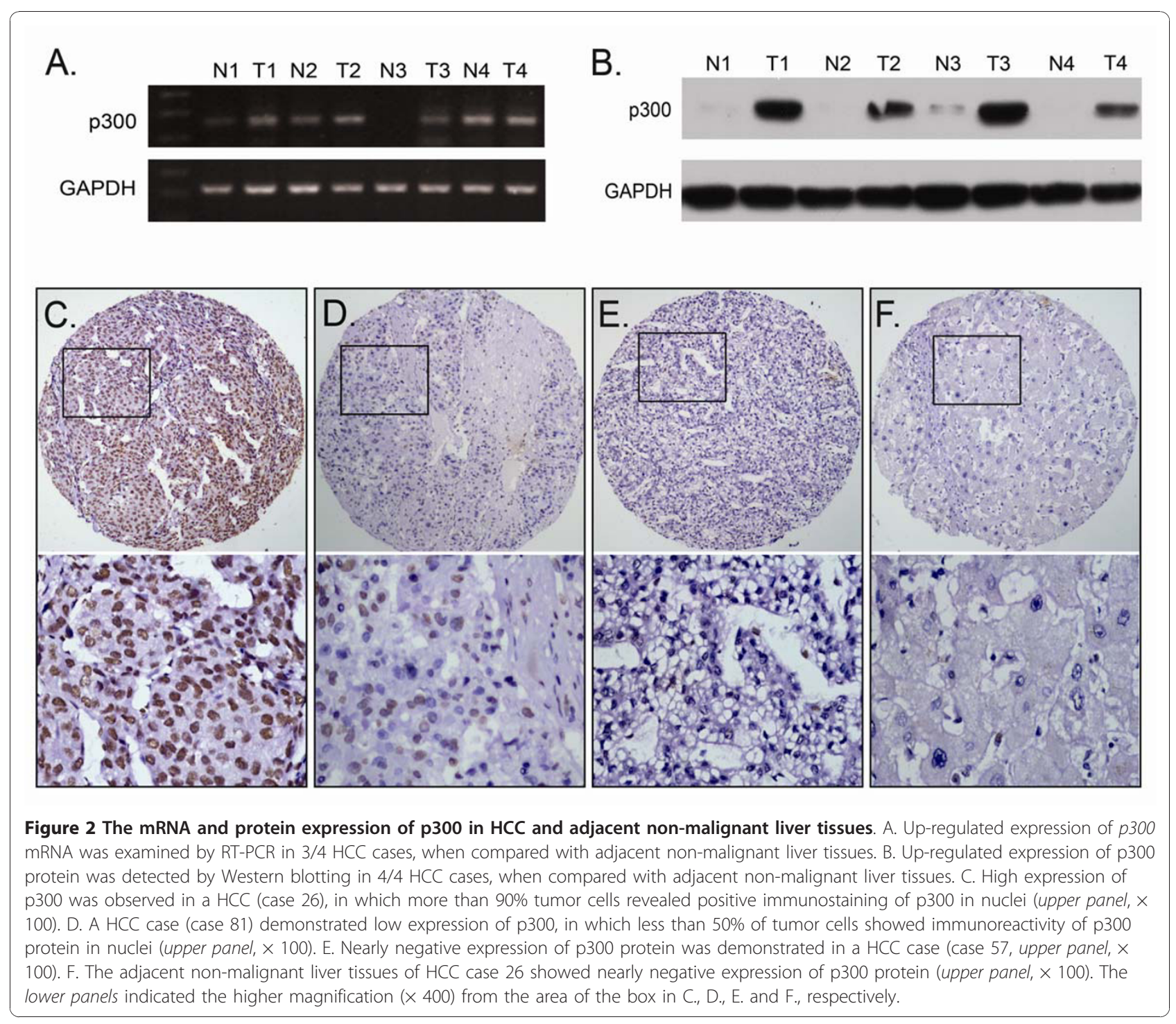

tissues (Figure 2A). Up-regulated expression of p300 protein was observed in $6 / 8(75.0 \%) \mathrm{HCCs}$, and in each of the four cases with up-regulated p300 protein, upregulated $p 300$ mRNA was observed (Figure $2 \mathrm{~B}$ ).

\section{The expression of p300 in HCC and adjacent non-} malignant liver tissues by IHC

For p300 IHC staining in HCCs and adjacent nonmalignant liver tissues, immunoreactivity was primarily observed in the nuclei within tumor cells (Figure 2C). p300 expression could be evaluated informatively in 123 HCCs by the TMA constructed previously. The noninformative 3 TMA samples included samples with too few tumor cells $(<300$ cells per case) and lost samples. Immunoreactivity of $\mathrm{p} 300$ in $\mathrm{HCC}$ ranged from $0 \%$ to $100 \%$ (Figure 2C-2F). According to ROC curve analysis, expression percentage for $\mathrm{p} 300$ above the cutoff value
$60 \%$ was defined as high expression, while below or equal to the cutoff value was considered as low expression. In this study, 16 of the 123 (13.0\%) HCC samples showed completely negative staining of p300. High expression of p300 could be detected in 60/123 (48.8\%) of HCCs, in 6/87 (6.9\%) of adjacent liver tissues with cirrhosis and in 2/36 (5.6\%) of adjacent normal liver tissues without cirrhosis, respectively $(P<0.0001$, Fisher's exact test).

\section{Selection of cutoff scores for $\mathrm{p} 300$ expression}

The ROC curves for each clinicopathological parameter (Figure 1) clearly show the point on the curve closest to $(0.0,1.0)$ which maximizes both sensitivity and specificity for the outcome as described in our previous study [19]. Tumors with scores above the obtained cutoff value were considered as high p300 expression leading 
Table 2 Area under the curve (AUC) of receiver operating characteristic curve for each clinicopathologic feature

\begin{tabular}{ccc}
\hline Variable & AUC $(\mathbf{9 5 \%} \mathbf{C l})$ & $\boldsymbol{P}$ value \\
\hline AFP & $0.662(0.563$ to 0.760$)$ & 0.002 \\
Tumor size & $0.703(0.606$ to 0.800$)$ & 0.000 \\
Tumor multiplicity & $0.633(0.525$ to 0.741$)$ & 0.019 \\
Differentiation & $0.634(0.536$ to 0.732$)$ & 0.010 \\
Stage & $0.609(0.505$ to 0.713$)$ & 0.044 \\
Vascular invasion & $0.544(0.441$ to 0.647$)$ & 0.407 \\
Relapse & $0.466(0.357$ to 0.576$)$ & 0.543 \\
\hline
\end{tabular}

$\mathrm{Cl}$ indicates confidence interval.

to the greatest number of tumors classified based on clinical outcome presence or absence. The corresponding area under the curve (AUC, 95\% CI) were collected and listed in Table 2. Cutoff score for p300 high expression was determined to be more than $60 \%$ carcinoma cells staining.

\section{Association of p300 expression with HCC patients' clinicopathological parameters}

The high or low expression rates of p300 in HCCs with respect to several standard clinicopathologic features are presented in Table 1. The high p300 expression rate was higher in patients with higher AFP levels $(P<0.0001)$, larger tumor size $(P<0.0001)$, tumor multiplicity $(P=$ $0.012)$, poorer differentiation $(P=0.036$, Table 1 , Figure 3 ) and later stage $(P=0.015$, Table 1$)$. There was no significant correlation between p300 expression and other clinicopathologic parameters, such as patient age $(\leq 47.7$ years $v s>47.7$ years), sex, hepatitis history, liver cirrhosis, tumor vascular invasion and relapse $(P>0.05$, Table 1$)$.

\section{Relationship between clinicopathologic features, p300 expression, and HCC patients' survival: Univariate survival analysis}

In order to confirm the representativeness of the HCCs in our study, we analyzed established prognostic factors of patients' survival. Kaplan-Meier analysis demonstrated a significant impact of well-known clinicopathologic prognostic parameters, such as serum AFP levels $(P<$ $0.0001)$, tumor size $(P<0.0001)$, tumor multiplicity $(P<$ $0.0001)$, clinical stage $(P<0.0001)$, vascular invasion $(P$ $<0.0001)$, and relapse $(P<0.0001)$ on patients' survival (Table 3). Assessment of survival in total HCCs revealed that high expression of p300 was correlated with adverse disease-specific survival of HCC patients $(P=0.001$, Table 3, Figure 4A). Further analysis was performed with regard to p300 expression in subsets of patients with different stages. The results demonstrated as well that high expression of p300 was a prognostic factor in HCC patients with stage II $(P=0.007$, Figure $4 \mathrm{~B})$ and stage III $(P=0.011$, Figure $4 C)$. However, it could not differentiate the outcome of stage I (not reached) or stage IV patients $(P=0.166$, Figure 4D).

\section{Independent prognostic factors of HCC: Multivariate Cox regression analysis}

Since features observed to have a prognostic influence by univariate analysis may covariate, p300 expression and those clinicopathologic variables that were significant in univariate analysis (i.e., AFP levels, tumor size, tumor multiplicity, clinical stage, vascular invasion, and relapse) were further examined in multivariate analysis. Results showed that high expression of p300 was an independent prognostic factor for poor patient overall survival (hazard ratio, 2.077; 95\%CI, 1.149-4.112, $P=$ 0.021; Table 3). Of the other parameters, serum AFP level $(P=0.014)$ and vascular invasion $(P=0.015)$ were evaluated as well independent prognostic factors for patients' overall survival.

\section{Prognostic model with p300 expression, AFP level and vascular invasion}

According to the results of our univariate and multivariate analyses, we proposed a new clinicopathologic prognostic model with three poor prognostic factors: p300 expression, AFP level and vascular invasion. Thus, we designated a high-risk group as the presence of the three factors (including p300 expression, AFP level and vascular invasion), an intermediate-risk group as the presence of two factor (regardless of their identity), and a low-risk group as the presence of one factor or none. The model could significantly stratify risk (low, intermediate and high) for overall survival based upon a combination of p300 and the standard clinicopathologic features $(P<0.0001$, Figure $4 \mathrm{E})$. In addition, application of Harrell concordance index to the proposed new clinicopathologic prognostic model showed improved predictive ability when compared with the standard pathological feature model (c indexes of 0.689 vs 0.648 , respectively).

\section{Correlation between p300 expression and cell proliferation in HCCs}

To address whether or not p300 expression in HCC is correlated with cell proliferation, the expression of $\mathrm{Ki}$ 67, a widely used cellular proliferation marker, was investigated by IHC in our HCC cohort. Among the 123 HCCs, in 118 samples, p300 and Ki-67 IHC were examined successfully and simultaneously. According to the ROC curve analysis, the cutoff score for Ki67 high expression was determined to be more than $50 \%$ carcinoma cells staining (data not shown). Using this designation, high expression of Ki67 was detected in 50/118 (42.4\%) HCCs. In addition, a significant positive correlation between expression of p300 and Ki67 was evaluated 

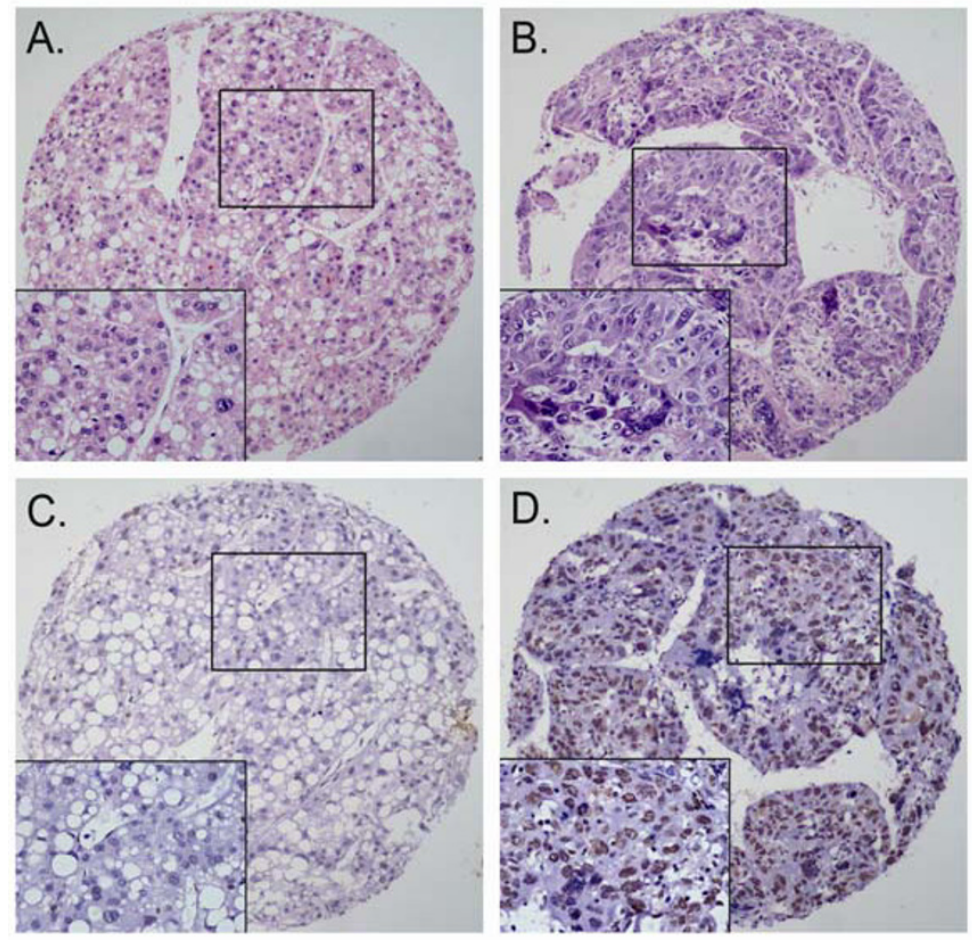

Figure 3 The altered expression levels of p300 in HCC tissues by immunohistochemistry. A. and B. represented H\&E staining for welldifferentiated HCC (case 43) and poorly-differentiated HCC (case 37), respectively. C. Low expression of p300 was observed in a welldifferentiated HCC case (case 43), in which less than $5 \%$ of tumor cells showed immunoreactivity of p300 protein in nuclei $(\times 100)$. D. High expression of p300 was demonstrated in the poor-differentiated HCC case (case 37), in which more than 60\% carcinoma cells showed immunoreactivity of p300 in nuclei $(\times 100)$. Representative sites in HCC tissue with higher (inset, $\times 400)$ magnification were shown.

in our HCC cohort, in which the frequency of cases with high expression of Ki67 was significantly larger in carcinomas with a high expression of p300 (32/56 cases, $57.1 \%)$ than in those cases with a low expression of p300 (18/62 cases, $29.0 \%)(P=0.002$, Table 1$)$.

\section{Discussion}

Transcriptional coactivator $\mathrm{p} 300$ has the potential to participate in a variety of cellular functions, such as cell proliferation and differentiation, senescence and apoptosis [7]. Recently several studies have documented an involvement of p300 in oncogenic processes, such as lung, colon, prostate, breast cancer and leukemia [14,21-24]. However, the status of p300 and its potential prognostic impact on HCC have not been explored so far. In the present study, we examined the expression levels of $p 300$ mRNA and p300 protein in HCC tissues and adjacent non-malignant liver tissues, firstly by RTPCR and Western blotting. Our results established that up-regulated expression of $p 300$ mRNA and p300 protein was shown in the majority of HCCs, when compared with their adjacent non-malignant liver tissues. Subsequently, the expression dynamics of p300 protein was investigated by IHC, using a TMA containing HCC tissues and adjacent non-malignant liver tissues. Our IHC results demonstrated that high expression of p300 was more frequently observed in HCC tissues when compared to the adjacent liver tissues with or without cirrhosis. The expression of p300 in adjacent non-malignant liver tissues with or without cirrhosis was either absent or at low levels. In contrast, in large number of our HCC tissues, high expression of p300 was frequently observed. Previous studies also described that mutation in $p 300$ gene, accompanied by loss of the other allele, was observed in certain types of tumors, including colorectal, gastric and breast cancers $[8,9]$. In addition, the frequency of promoter methylation of $p 300$ gene was found in $65.8 \%$ of HCC [25]. These findings provide evidence that the up-regulation of p300 may play an important role in tumorigenic process of HCC.

To assess the significance of $\mathrm{p} 300$ protein in $\mathrm{HCC}$ and avoid predetermined arbitrary cutpoint, ROC curve analysis was applied to determine cutoff score for p300 expression as described in our previous study [19]. Further correlation analysis revealed that high expression of p300 in HCCs was correlated with higher serum AFP levels, larger tumor size, tumor multiplicity, poorer differentiation and later clinical stage. 
Table 3 Univariate and multivariate analysis of different prognostic factors in 123 patients with hepatocellular carcinoma (Cox Proportional Hazards Regression)

\begin{tabular}{|c|c|c|c|c|c|}
\hline \multirow[b]{2}{*}{ Variable } & \multicolumn{3}{|c|}{ Univariate analysis } & \multicolumn{2}{|c|}{ Multivariate analysis } \\
\hline & All cases & HR $(95 \% \mathrm{Cl})$ & $P$ value & HR $(95 \% \mathrm{Cl})$ & $P$ value \\
\hline Age (years) & & & 0.883 & & \\
\hline$\leq 47.9^{\mathrm{a}}$ & 59 & 1.0 & & & \\
\hline$>47.9$ & 64 & $1.044(0.588-1.853)$ & & & \\
\hline Sex & & & 0.746 & & \\
\hline Male & 107 & $1.153(0.489-2.717)$ & & & \\
\hline Female & 16 & 1.0 & & & \\
\hline Hepatitis history & & & 0.806 & & \\
\hline Yes & 105 & $0.904(0.405-2.021)$ & & & \\
\hline No & 18 & 1.0 & & & \\
\hline AFP $(n g / m l)$ & & & 0.000 & & 0.014 \\
\hline$\leq 20$ & 68 & 1.0 & & 1.0 & \\
\hline$>20$ & 55 & $5.445(2.852-10.395)$ & & $2.573(1.209-5.476)$ & \\
\hline Liver cirrhosis & & & 0.807 & & \\
\hline Yes & 87 & 1.0 & & & \\
\hline No & 36 & $1.082(0.578-2.026)$ & & & \\
\hline Tumor size $(\mathrm{cm})$ & & & 0.000 & & 0.167 \\
\hline$\leq 5$ & 76 & 1.0 & & 1.0 & \\
\hline$>5$ & 47 & $2.946(1.640-5.290)$ & & 1.595 (0.823-3.090) & \\
\hline Tumor multiplicity & & & 0.000 & & 0.077 \\
\hline Single & 85 & 1.0 & & 1.0 & \\
\hline Multiple & 38 & $3.768(2.108-6.735)$ & & 1.790 (0.939-3.414) & \\
\hline Differentiation & & & 0.099 & & \\
\hline Well-moderate & 85 & 1.0 & & & \\
\hline Poor-undifferentiated & 38 & $1.642(0.911-2.958)$ & & & \\
\hline Stage & & & 0.000 & & 0.363 \\
\hline$|-| \mid$ & 61 & 1.0 & & 1.0 & \\
\hline III -IV & 62 & $5.828(2.722-12.480)$ & & $1.571(0.593-4.162)$ & \\
\hline Vascular invasion & & & 0.000 & & 0.015 \\
\hline Yes & 55 & $5.372(2.724-10.595)$ & & $2.724(1.214-6.113)$ & \\
\hline No & 68 & 1.0 & & 1.0 & \\
\hline Relapse & & & 0.000 & & 0.321 \\
\hline Yes & 42 & $2.885(1.608-5.174)$ & & $1.390(0.725-2.666)$ & \\
\hline No & 81 & 1.0 & & 1.0 & \\
\hline p300 & & & 0.001 & & 0.021 \\
\hline Low expression & 63 & 1.0 & & 1.0 & \\
\hline High expression & 60 & $2.792(1.533-5.087)$ & & 2.077 (1.149-4.112) & \\
\hline Ki67 & & & 0.089 & & \\
\hline Low expression & 68 & 1.0 & & & \\
\hline High expression & 50 & $1.661(0.925-2.982)$ & & & \\
\hline
\end{tabular}

a Mean age; AFP, alpha-fetoprotein; HR, hazards ratio; $\mathrm{Cl}$, confidence interval.

Importantly, high expression of p300 was a strong and independent predictor of shortened overall survival as evidenced by univariate and multivariate analysis. In addition, stratified survival analysis of HCC accordingly to clinical stage evaluated p300 expression to be closely correlated with survival of HCC patients with stage II or stage III. Since a relatively less cases of HCC were included in stage I or stage IV, we did not found statistically significant correlation for these HCC-subgroups in univariate analysis. Our findings in this study suggest that expression of p300 in HCC may facilitate an increased malignant feature and/or worse prognosis of this tumor. Previous study also suggested that putative p300 and CREB complex might up-regulate the $\mathrm{H} 3$ and H4 acetylation levels, and then up-regulated the Hulc expression level which was identified as the 
A.

Total

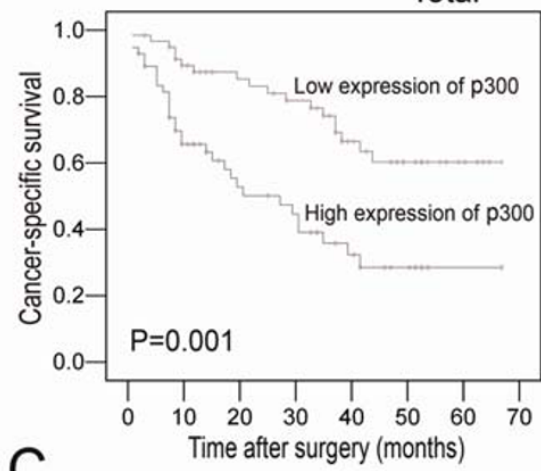

C.

Stage III

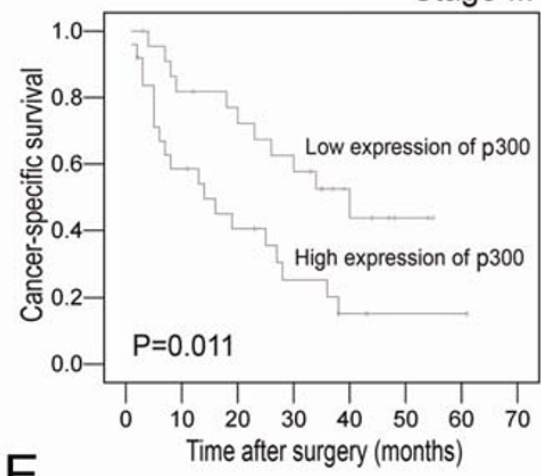

E.

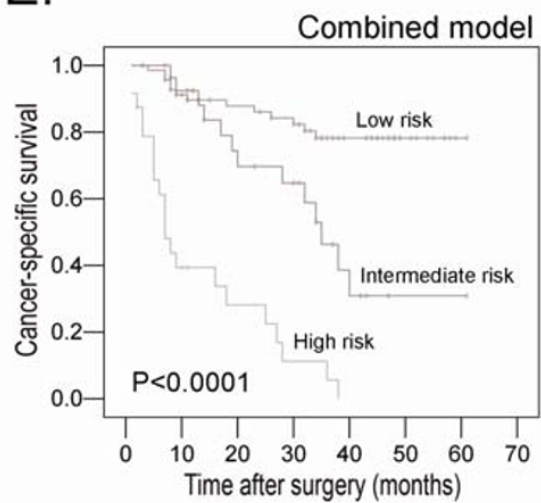

B.
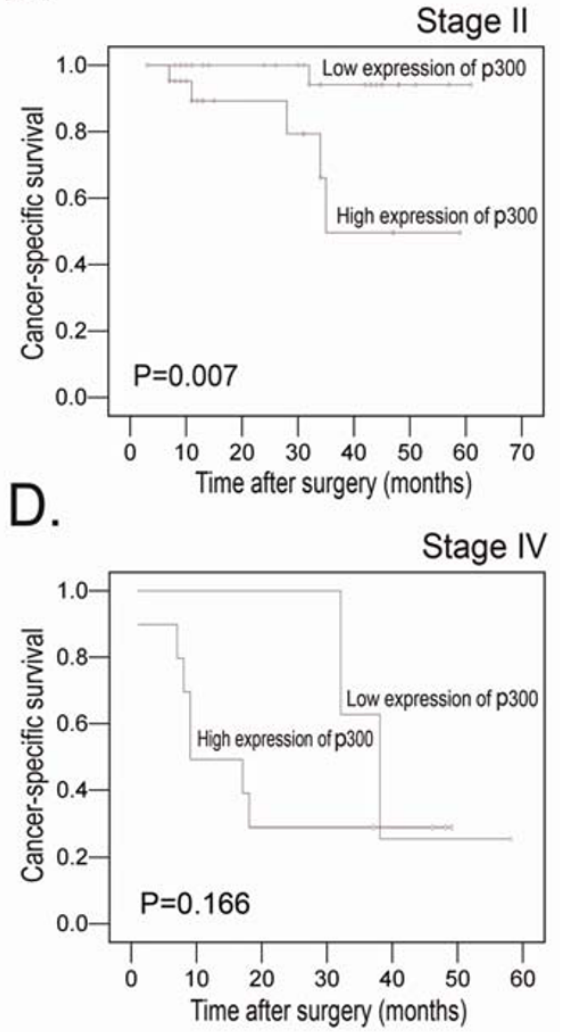

Figure 4 Kaplan-Meier survival analysis of p300 expression in total patients and subsets of different stage patients with HCC (log-rank test). A. Total, probability of survival of all patients with HCC: low expression, $n=63$; high expression, $n=60$. B. Stage II, probability of survival of stage II patients with HCC: low expression, $n=27$; high expression, $n=22$. C. Stage III, probability of survival of stage III patients with HCC: low expression, $n=23$; high expression, $n=25$. D. Stage $N$, probability of survival of stage IV patients with HCC: low expression, $n=3$; high expression, $n=11$. E. Comparison of overall survival according to a new combined clinicopathologic prognostic model (including p300, AFP level and vascular invasion): low risk, $n=70$; intermediate risk, $n=29$; high risk, $n=24$.

most important genes in HCC [13]. Thus, the examination of p300 expression by IHC could be used as an additional tool in identifying those patients at risk of HCC progression; p300 expression analysis may also be useful in optimizing individual HCC therapy management: favoring a more aggressive regimen in tumors with a high expression of $\mathrm{p} 300$.
Although several characteristics of CBP and p300 suggested that these proteins might serve as tumor suppressors, some studies reported an important role of p300 protein in oncogenic processes [7,26]. In prostate cancer, p300 expression was shown to be linked to proliferation and identified as a predictor of progression of this cancer [14]. In colon carcinoma, overexpression 
of p300 was an indicator of poor prognosis [21]. Moreover, p300 mRNA levels were observed to correlate with lymph node status in breast cancer [24]. However, p300 protein levels did not show significant correlations with tumor grade or nodal positivity in other study [27,28]. In the present study, we did observe that high expression of p300 was associated with an aggressive feature of HCC and was a strong and independent predictor of shorter cancer-specific survival. Considering that the mechanism by which coactivator $\mathrm{p} 300$ promotes gene transcription may vary among gene targets, it is not very difficult for us to understand that the function of p300 and its underling mechanism(s) to impact cancer progression may lead to this discrepancy. In addition, although we observed a positive association of p300 expression and Ki-67 expression (a marker for cell proliferation) in our HCC cohort, the precise signaling pathway that is ultimately involved in these processes remains to be investigated. However, our findings suggest a potential important role of $p 300$ in the control of HCC cell proliferation, an activity that might be responsible, at least in part, for HCC tumorigenesis and/or progression.

Since advanced pTNM stage and tumor differentiation are the best-established risk factors for important aspects affecting the prognosis of patients with HCC [29]. These 2 parameters, based on specific clinicopathologic features and extent of disease, may have reached their limits in providing critical information influencing patient prognosis and treatment strategies. Furthermore, outcome of patients with same stage following surgery is substantially different and such large discrepancy has not been explored [30,31]. Thus, there is a need for new objective strategies that can effectively distinguish between patients with favorable and unfavorable prognosis. In this study, our results support the ideas that p300 expression, as examined by IHC, can identify patients with HCC that may show aggressive clinical course and poor outcome. Therefore, evaluation of p300 expression may become a biomarker for predicting prognosis and rendering a more tailored treatment strategy in patients with HCC. Based on the results, we propose a new prognostic model with high p300 expression, AFP levels and vascular invasion. This model including p300 expression can reflect the aggressive phenotype of HCC. Furthermore, its prognostic significance can be augmented by the elevated AFP levels and the presence of vascular invasion. Thus, this combined model may be a useful prognostic index for HCC.

\section{Conclusions}

Our findings provide a basis for the concept that high expression of p300 may play an important role in the acquisition of an aggressive phenotype in HCC, suggesting that the expression of p300, as examined by IHC, will be a promising independent biomarker for shortened survival time of HCC patients. The combined clinicopathologic prognostic model may become a useful tool for identifying patients with different clinical outcomes.

\section{Abbreviations}

AFP: alpha-fetoprotein; AUC: area under the curve; CBP: CREB binding protein; CREB: CAMP response element binding protein; HCC: hepatocellular carcinoma; Hulc: highly up-regulated in liver cancer; $\mathrm{HC}$ : immunohistochemistry; ROC: receiver operating characteristic; TMA: tissue microarray.

\section{Acknowledgements}

This study was supported by grant from the Nature Science Foundation of China (No.30901709).

\section{Author details}

'State Key Laboratory of Oncology in South China, Sun Yat-Sen University Cancer Center, Guangzhou, PR China. ²Department of Pathology, Sun YatSen University Cancer Center, Guangzhou, PR China.

\section{Authors' contributions}

MYC is responsible for the study design. ML and RZL performed the experiments and draft the manuscript. JWC, JBL YC, JHH and QLW participated in the data analysis and interpretation. All authors read and approved the final manuscipt.

\section{Competing interests}

The authors declare that they have no competing interests.

Received: 24 September 2010 Accepted: 5 January 2011

Published: 5 January 2011

\section{References}

1. Lau WY, Lai EC, Lau SH: The current role of neoadjuvant/adjuvant/ chemoprevention therapy in partial hepatectomy for hepatocellular carcinoma: a systematic review. Hepatobiliary Pancreat Dis Int 2009, 8:124-133.

2. Jemal A, Siegel R, Ward E, Hao Y, Xu J, Thun MJ: Cancer statistics, 2009. CA Cancer J Clin 2009, 59:225-249.

3. Cabibbo G, Craxi A: Epidemiology, risk factors and surveillance of hepatocellular carcinoma. Eur Rev Med Pharmacol Sci 14:352-355.

4. Frau M, Biasi F, Feo F, Pascale RM: Prognostic markers and putative therapeutic targets for hepatocellular carcinoma. Mol Aspects Med 31:179-193.

5. Kundu TK, Palhan VB, Wang Z, An W, Cole PA, Roeder RG: Activatordependent transcription from chromatin in vitro involving targeted histone acetylation by p300. Mol Cell 2000, 6:551-561.

6. Vo N, Goodman RH: CREB-binding protein and p300 in transcriptional regulation. J Biol Chem 2001, 276:13505-13508.

7. Goodman RH, Smolik S: CBP/p300 in cell growth, transformation, and development. Genes Dev 2000, 14:1553-1577.

8. Muraoka M, Konishi M, Kikuchi-Yanoshita R, Tanaka K, Shitara N, Chong JM, Iwama T, Miyaki M: p300 gene alterations in colorectal and gastric carcinomas. Oncogene 1996, 12:1565-1569.

9. Gayther SA, Batley SJ, Linger L, Bannister A, Thorpe K, Chin SF, Daigo Y, Russell P, Wilson A, Sowter HM, et al: Mutations truncating the EP300 acetylase in human cancers. Nat Genet 2000, 24:300-303.

10. Fan $\mathrm{S}, \mathrm{Ma} Y \mathrm{YX}$, Wang $\mathrm{C}$, Yuan $\mathrm{RQ}$, Meng Q, Wang JA, Erdos M, Goldberg ID, Webb P, Kushner PJ, et al: p300 Modulates the BRCA1 inhibition of estrogen receptor activity. Cancer Res 2002, 62:141-151.

11. Bandyopadhyay D, Okan NA, Bales E, Nascimento L, Cole PA, Medrano EE: Down-regulation of $\mathrm{p} 300 / \mathrm{CBP}$ histone acetyltransferase activates a senescence checkpoint in human melanocytes. Cancer Res 2002. 62:6231-6239. 
12. Fang Z, Fu Y, Liang Y, Li Z, Zhang W, Jin J, Yang Y, Zha X: Increased expression of integrin beta1 subunit enhances p21WAF1/Cip1 transcription through the Sp1 sites and p300-mediated histone acetylation in human hepatocellular carcinoma cells. J Cell Biochem 2007, 101:654-664

13. Wang J, Liu X, Wu H, Ni P, Gu Z, Qiao Y, Chen N, Sun F, Fan Q: CREB upregulates long non-coding RNA, HULC expression through interaction with microRNA-372 in liver cancer. Nucleic Acids Res 38:5366-5383.

14. Debes JD, Sebo TJ, Lohse CM, Murphy LM, Haugen DA, Tindall DJ: p300 in prostate cancer proliferation and progression. Cancer Res 2003, 63:7638-7640.

15. Isharwal S, Miller MC, Marlow C, Makarov DV, Partin AW, Veltri RW: p300 (histone acetyltransferase) biomarker predicts prostate cancer biochemical recurrence and correlates with changes in epithelia nuclear size and shape. Prostate 2008, 68:1097-1104.

16. Gao Q, Qiu SJ, Fan J, Zhou J, Wang XY, Xiao YS, Xu Y, Li YW, Tang ZY: Intratumoral balance of regulatory and cytotoxic $\mathrm{T}$ cells is associated with prognosis of hepatocellular carcinoma after resection. J Clin Oncol 2007, 25:2586-2593.

17. Sobin LH, Fleming ID: TNM Classification of Malignant Tumors, fifth edition (1997). Union Internationale Contre le Cancer and the American Joint Committee on Cancer. Cancer 1997, 80:1803-1804.

18. Han L, Lu J, Pan L, Wang X, Shao Y, Han S, Huang B: Histone acetyltransferase $p 300$ regulates the transcription of human erythroidspecific 5-aminolevulinate synthase gene. Biochem Biophys Res Commun 2006, 348:799-806

19. Cai MY, Zhang B, He WP, Yang GF, Rao HL, Rao ZY, Wu QL, Guan XY, Kung $H F$, Zeng $Y X$, Xie D: Decreased expression of PinX1 protein is correlated with tumor development and is a new independent poor prognostic factor in ovarian carcinoma. Cancer Sci 101:1543-1549.

20. Zlobec I, Steele R, Terracciano L, Jass JR, Lugli A: Selecting immunohistochemical cut-off scores for novel biomarkers of progression and survival in colorectal cancer. J Clin Pathol 2007, 60:1112-1116.

21. Ishihama K, Yamakawa M, Semba S, Takeda H, Kawata S, Kimura S, Kimura W: Expression of HDAC1 and CBP/p300 in human colorectal carcinomas. J Clin Pathol 2007, 60:1205-1210.

22. Karamouzis MV, Konstantinopoulos PA, Papavassiliou AG: Roles of CREBbinding protein $(\mathrm{CBP}) / \mathrm{p} 300$ in respiratory epithelium tumorigenesis. Cell Res 2007, 17:324-332

23. Borrow J, Stanton VP Jr, Andresen JM, Becher R, Behm FG, Chaganti RS, Civin Cl, Disteche C, Dube I, Frischauf AM, et al: The translocation $\mathrm{t}(8 ; 16)$ (p11;p13) of acute myeloid leukaemia fuses a putative acetyltransferase to the CREB-binding protein. Nat Genet 1996, 14:33-41.

24. Kurebayashi J, Otsuki T, Kunisue H, Tanaka K, Yamamoto S, Sonoo H: Expression levels of estrogen receptor-alpha, estrogen receptor-beta, coactivators, and corepressors in breast cancer. Clin Cancer Res 2000, 6:512-518.

25. Zhang C, Guo X, Jiang G, Zhang L, Yang Y, Shen F, Wu M, Wei L: CpG island methylator phenotype association with upregulated telomerase activity in hepatocellular carcinoma. Int J Cancer 2008, 123:998-1004.

26. Fermento ME, Gandini NA, Lang CA, Perez JE, Maturi HV, Curino AC, Facchinetti MM: Intracellular distribution of p300 and its differential recruitment to aggresomes in breast cancer. Exp Mol Pathol 88:256-264.

27. De-Carvalho MC, Chimelli LM, Quirico-Santos T: Modulation of fibronectin expression in the central nervous system of Lewis rats with experimental autoimmune encephalomyelitis. Braz J Med Biol Res 1999, 32:583-592.

28. Hudelist G, Czerwenka K, Kubista E, Marton E, Pischinger K, Singer CF: Expression of sex steroid receptors and their co-factors in normal and malignant breast tissue: AIB1 is a carcinoma-specific co-activator. Breast Cancer Res Treat 2003, 78:193-204.

29. Farinati F, Rinaldi M, Gianni S, Naccarato R: How should patients with hepatocellular carcinoma be staged? Validation of a new prognostic system. Cancer 2000, 89:2266-2273.

30. Lau WY, Lai EC: Hepatocellular carcinoma: current management and recent advances. Hepatobiliary Pancreat Dis Int 2008, 7:237-257.

31. Bruix J, Sherman M: Management of hepatocellular carcinoma. Hepatology 2005, 42:1208-1236.

\section{doi:10.1186/1479-5876-9-5}

Cite this article as: Li et al: High expression of transcriptional coactivator p300 correlates with aggressive features and poor prognosis of hepatocellular carcinoma. Journal of Translational Medicine 2011 9:5

\section{Submit your next manuscript to BioMed Central and take full advantage of:}

- Convenient online submission

- Thorough peer review

- No space constraints or color figure charges

- Immediate publication on acceptance

- Inclusion in PubMed, CAS, Scopus and Google Scholar

- Research which is freely available for redistribution

Submit your manuscript at www.biomedcentral.com/submit
Ciomed Central 